\title{
Orbital Cellulitis due to Pseudomonas aeruginosa in Renal Transplant Recipient
}

\author{
Khin Phyu Pyar*, Sai Aik Hla, Win Kyaw Shwe, Kyaw Thurein Lwin, Min Aung Hein, Lay Maung Maung \\ and Chan Aye Aung \\ Professor/Consultant Physician \& Head of Clinical Wing, Myanmar \\ *Corresponding author: Khin Phyu Pyar, Professor/Consultant Physician \& Head of Clinical Wing, Myanmar
}

\begin{tabular}{|c|c|}
\hline ARTICLE INFO & ABSTRACT \\
\hline Received: 輊 February 14, 2019 & Summary \\
\hline Published: 幽 February 25, 2019 & $\begin{array}{l}\text { A } 43 \text { year old renal transplant recipient had severe headache, fever and proptosis on } \\
7 \text { post- operative day. MRI head revealed oedematous orbit with opaque maxillary and }\end{array}$ \\
\hline $\begin{array}{l}\text { Citation: Khin Phyu P, Sai Aik H, Win } \\
\text { Kyaw S, Kyaw Thurein L, Min Aung H, } \\
\text { Lay Maung M, Chan Aye A. Orbital Cel- } \\
\text { lulitis due to Pseudomonas aeruginosa }\end{array}$ & $\begin{array}{l}\text { ethmoid sinuses suggestive of sinusitis on same side. The culture of antrum wash out fluid } \\
\text { from maxillary sinus e showed the growth of Pseudomonas aeruginosa. It was resistant } \\
\text { to all antibiotics except gentamycin and amikacin. The patient expired } 3 \text { weeks after } \\
\text { receiving new kidney. }\end{array}$ \\
\hline
\end{tabular}

in Renal Transplant Recipient. Biomed J Sci \& Tech Res 15(1)-2019. BJSTR. MS.ID.002649.

Keywords: Renal Transplant Recipient; Orbital Cellulitis; Sinusitis; Pseudomonas aeruginosa

\section{Introduction}

In the first month post-transplantation period, the degree of immunosuppression is not high enough to get opportunistic infections. Thus, the most frequent infections are related to surgical and nosocomial complications. The common infections are bacterial and candidal wound infections, urinary tract infections, nosocomial pneumonias, and central venous catheter-associated bacteremias and fungemias. The orbital cellulitis due to Pseudomonas aeruginosa was reported in some immune-compromised patients. One report mentioned ecthyma gangrenosum -focal skin necrosis in orbital cellulitis was associated with septicaemia and death.

\section{Case Presentation}

The patient was 43 year old, a case of end stage renal disease due to diabetes mellitus. He had maintained on haemodialysis two times a week for 3 years while he was waiting for viral clearance for hepatitis B viral infection. He got antiviral therapy supervised by hepatologist. When his viral load was undetectable, he underwent transplant, donated from his brother. His brother also had hepatitis B viral infection and thus, he took treatment. The timing for transplant was planned only when the viral load of the donor was undetectable. The patient was still on Entecavir. The HLA matching was $100 \%$ compatible and CDC cross match was negative. The transplant team decided to give lower dose of immunosuppressant as their HLA matching was excellent.

The immediate post-operative period was uneventful (Figure 1). He was on methyl prednisolone, tacrolimus, mycophenolate mofetil, trimethoprim- sulphamethoxazole, nystatin and cefopyrazonesulbactam according to our transplant protocol. The blood level of tacrolimus was monitored at 48 hour and we kept the level at lower side. His glycaemic control was okay. After 3 days intensive care at ICU, he was shifted to renal unit.

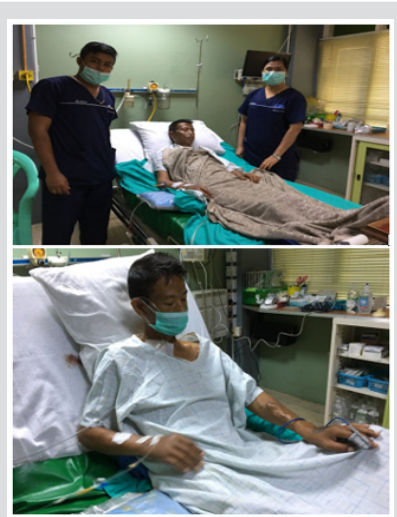

Figure 1. 
Three days later, he had severe headache and fever. There was exopthalmos, chemosis and external opthalmoplegia on tenth post-transplant day (Figure 2). He had complete opthalmoplegia with pupil dilatation on left side (Figure 3). The vision was near normal. The eye was not pulsatile, and bruit was not detected. Orbital cellulitis due to bacteria was considered and thus we gave imipenum and metronidazole after taking blood culture. The dosage of tacrolimus and mycophenolate mofetil were reduced to improve his immunity.

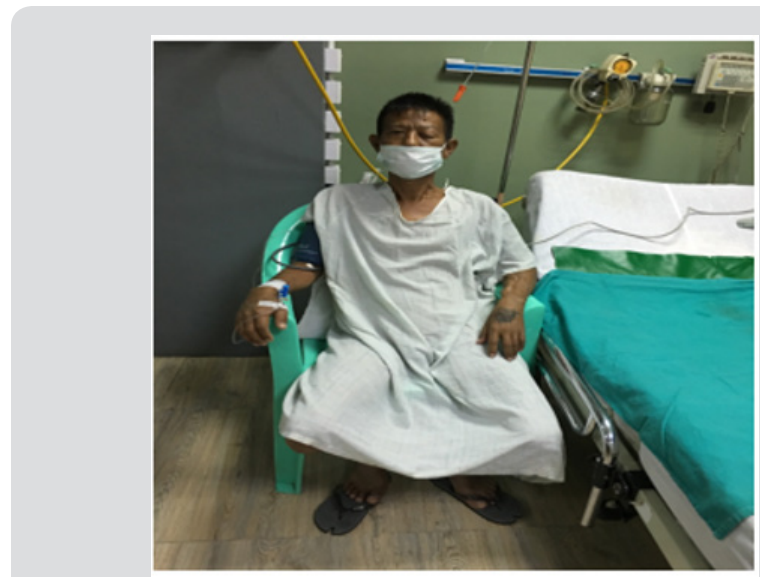

Figure 2.

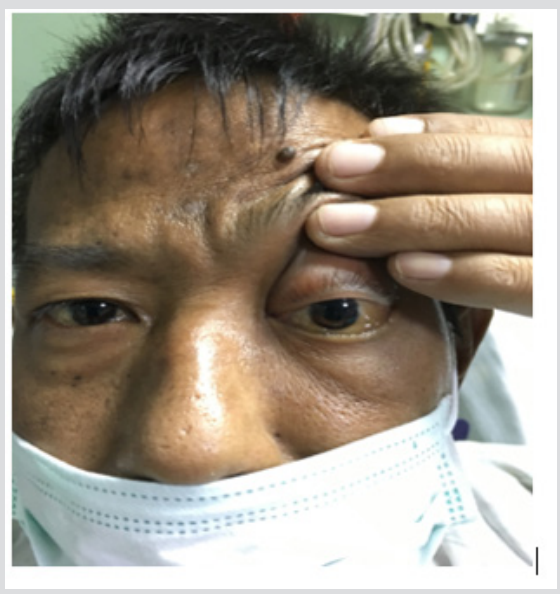

Figure 3.

His total WBC count rose to $17.8 \times 109 / 1$ ( immediate post op day 1 was 7.4 X 109/l) with neutrophilla ( 95\%). CT scan revealed ethmoid sinusitis and oedematus orbital muscle and fat (Figures 4 \& 5). The possibility of cavernous sinus thrombosis due to infective aetiology was considered. Cerebral MRI /MRA/MRV was normal, thus excluding of venous sinus thrombosis (Figures $6 \&$ 7). MRI showed proptosis, swollen orbit, opaque maxillary and ethmoid sinuses (Figures 7 \& 8) The ophthalmologist agreed same antibiotics. His sinuses were very congested and oedematous with purulent exudate on nasal endoscopic examination. Antrum wash out was done and the fluid was sent for culture.

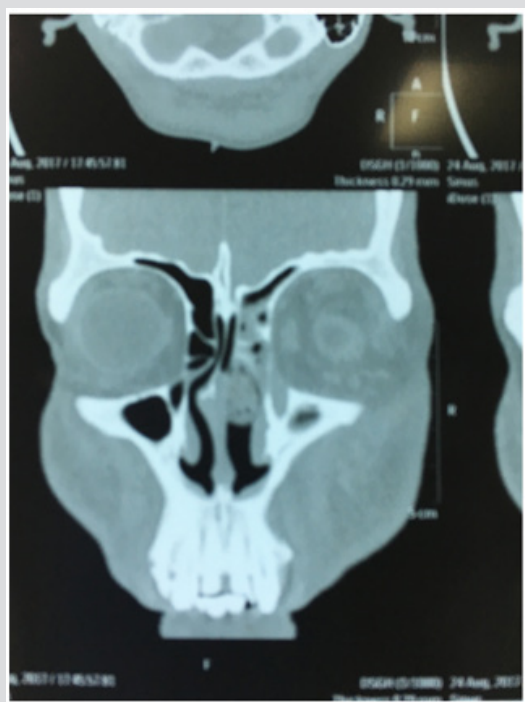

Figure 4.

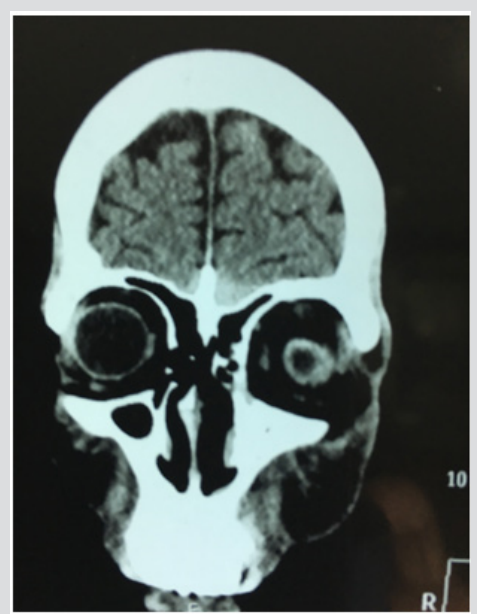

Figure 5.

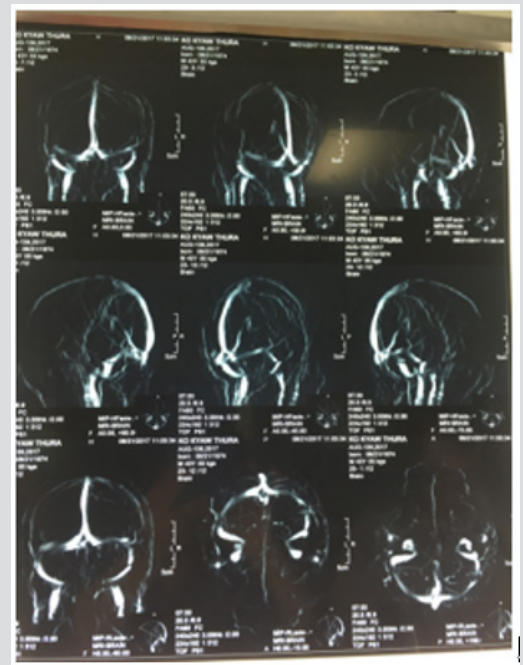

Figure 6. 

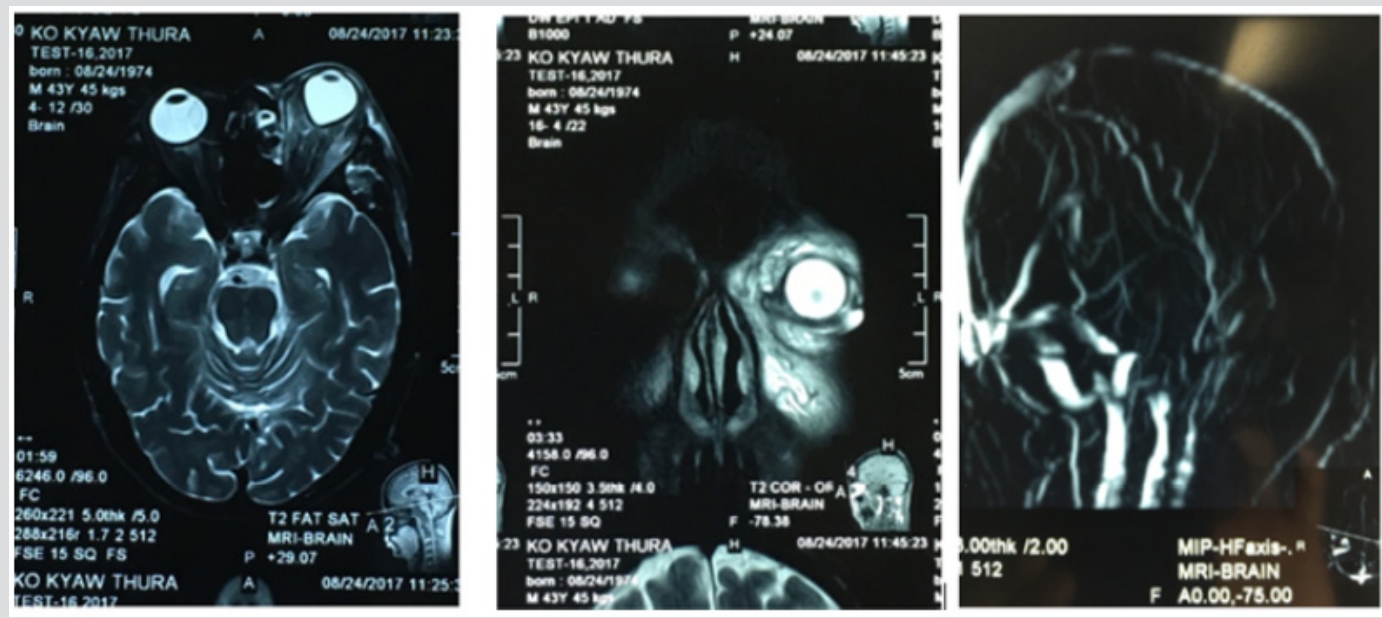

Figure 7.
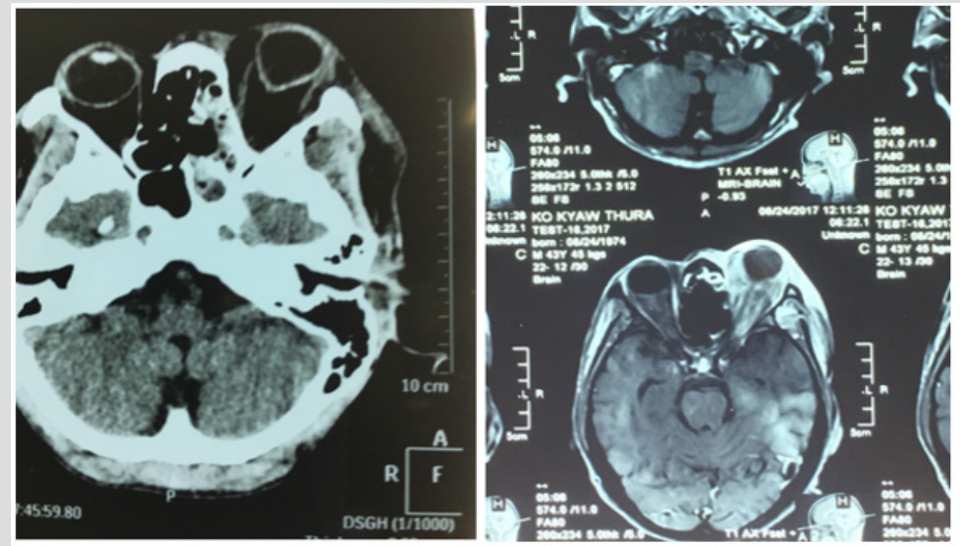

Figure 8.

His fever was swinging. The proptosis was increasing, and conjunctival oedema became worse (Figure 9) He was drowsy. There was focal skin necrosis nearby (Ecthyma gangrenosum) (Figure 10). His general condition became more deteriorated, blood pressure dropped. Blood culture was sterile. The culture from the fluid
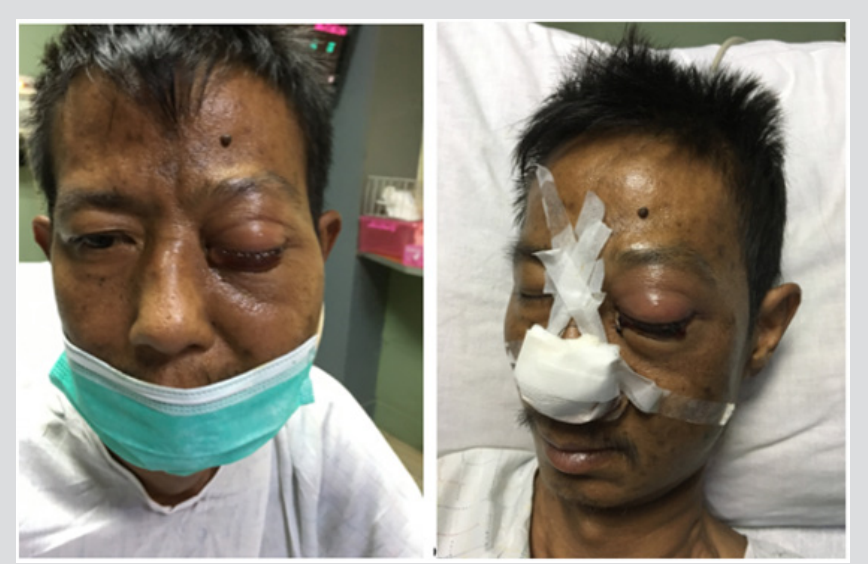

Figure 9. from maxillary sinus showed growth of Pseudomonas aeruginosa (Figure 11) It was resistant to all antibiotics except gentamycin and amikacin. The MIC was (1) and (2) respectively. Thus, injection amikacin was added. He expired 3 weeks after receiving new kidney.

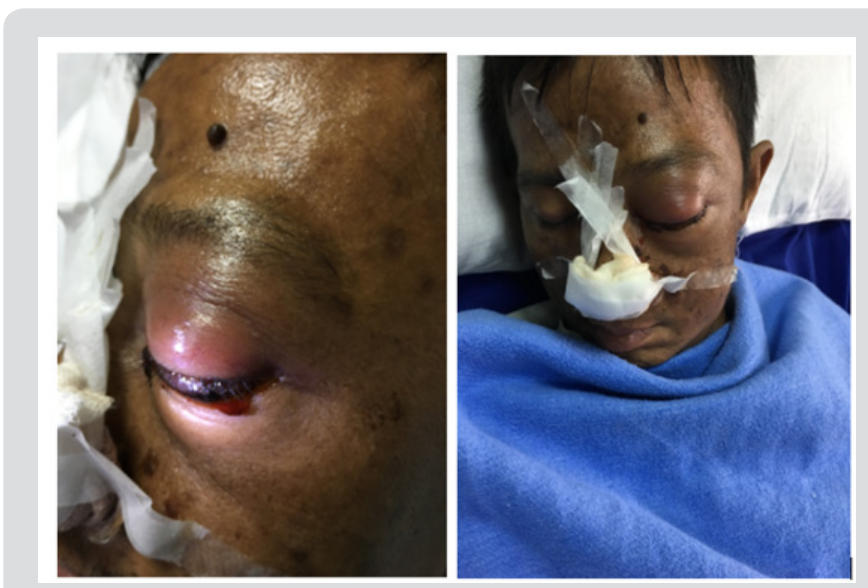

Figure 10. 


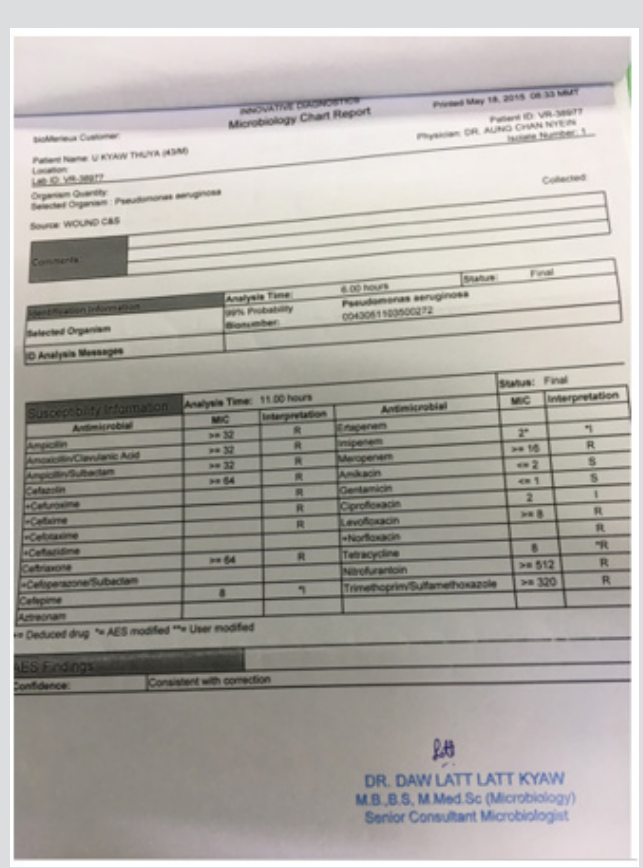

Figure 11.

\section{Discussion}

The living kidney donor transplants have been done in No. (1) Defence Services General Hospital, Myanmar since 1995. We have 210 cases till now. One of the common infections was urinary tract infection. It was noted in $6 \%$ of them and Escheria coli, Pseudomonas aeruginosa and Candida albicans were grown in urine culture. The central venous catheter infection was seen in 5\% of recipients and their main organisms were Staphylococcus aureus, Pseudomonas aeruginosa and Burkholderia cepacia. Among them 5 cases expired one month after transplant period. This case was one of them.

Regarding the timing of infection and immunosuppression, the infection in first month post renal transplant was due surgical and nosocomial complications. The immune system was not too suppressed in initial weeks. If a recipient had surgical complication, he had more chances of developing infection. Disseminated mucomycosis leading to death in post- transplant one month was reported in one patient with surgical complication [1]. As our patient did not have surgical complication, he probably acquired infection from re- nal unit, not from ICU or operating room. In sino-orbital infection, Pseudomonas aeruginosa was the main organism [2]. In immunocompromised state, it could lead to severe orbital cellulitis and septicaemia $[3,4]$ Our patient had similar picture like them.

Preseptal orbital cellulitis and focal necrosis (ecthyma gangrenosum) in a patient with severe immunocompromised was reported in 2009 [5] And it was due to Pseudomonas aeruginosa. It was associated with septicaemis and death. Bur patient had focal necrosis around nose, but erythema was not prominent as his skin colour was brown.

Pseudomonas aeruginosa was known to have antimicrobial resistance and commonly led to septicaemia and multi-organ failure even in immune-competent host. This patient was resistant to nearly all antibiotics except aminoglycoside which were barely sensitive (MIC 1-2). We gave imepenum and it was resistant. One study done in tertiary hospital revealed that $30-40 \%$ of isolates of Pseudomonas aeruginosa were resistant to meropenum and imipenum [6]. Their study also pointed out growing anti-microbial resistance of it. Thus, combination of immunocompromised state (immunosuppressants and known diabetes mellitus) and virulent Pseudomonas aeruginosa infection led to septicaemia and death in our patient.

\section{References}

1. Hamdi T, Karthikeyan V, George J Alangaden GJ (2014) Mucormycosis in a renal transplant recipient: Case report and comprehensive review of literature. Int J Nephrol 214: 950643.

2. Chen X, Bleier BS, Lefebvre DR, Lee NG (2016) Pseudomonas aeruginosa: A masquerader in sino-orbital infections. Ophthalmic Plast Reconstr Surg 32(5): 374-377.

3. Cano-Parra J, Espafia E, Esteban M, Diaz-Llopis M, Menezo JL (1994) Pseudomonas conjunctival ulcer and secondary orbital cellulitis in a patient with AIDS. Br J Ophthalmol 78: 72-73.

4. Singh I, Gupta V, Gupta SK, Anubhav S (2017) Acute orbital cellulitis: A case series. Glob J Oto 3(3): 555614.

5. Edward A Hulten, Anita A Shah, Kyle N Petersen, Christopher M Gallagher, Peter H Vangeertruyden (2009) Pseudomonas aeruginosa preseptal cellulitis and focal necrosis in a patient with severe immunocompromised. Infectious Diseases in Clinical Practice 17(5): 346-348.

6. Lila G, Mulliqi-Osmani G, Bajrami R, Kurti A, Azizi E, et al. (2017) The prevalence and resistance patterns of Pseudomonas aeruginosa in a tertiary care hospital in Kosovo. Infez Med 25(1): 21-26.

\section{ISSN: 2574-1241}

DOI: 10.26717/BJSTR.2019.15.002649

Khin Phyu Pyar. Biomed J Sci \& Tech Res

(C) This work is licensed under Creative

Submission Link: https://biomedres.us/submit-manuscript.php

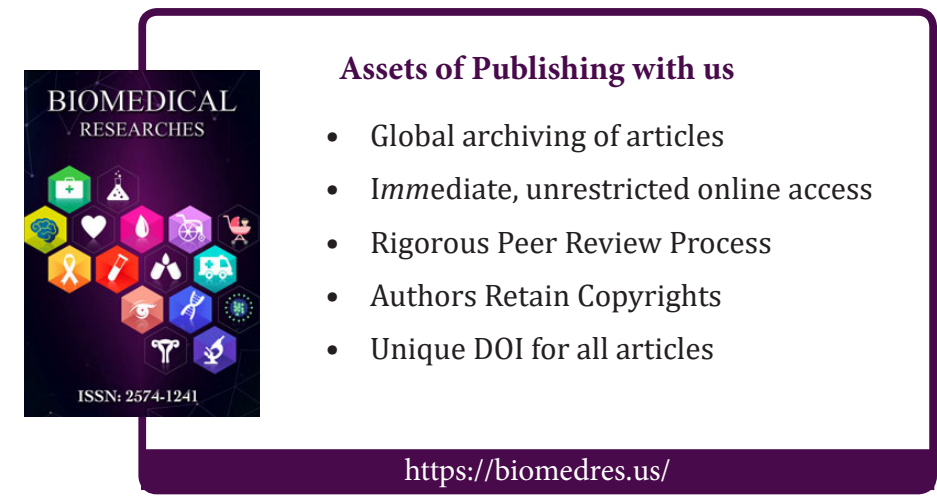

